\title{
Dynamic Bandwidth Management in IEEE 802.11-Based Multihop Wireless Networks
}

\author{
Cheikh Sarr • Sofiane Khalfallah • Isabelle \\ Guérin-Lassous
}

Received: date / Accepted: date

\begin{abstract}
In this paper, we propose a new protocol named DRBT (Dynamic Regulation of Best Effort Traffic) which supports QoS throughput guarantees and provides a distributed regulation mechanism for Best Effort traffic in multihop wireless networks. By adapting dynamically the rate of Best Effort traffic at the link layer, DRBT increases the acceptance ratio of QoS flows and provides a good use of the remaining resources through the network. Our protocol also provides an accurate method to evaluate the available bandwidth in IEEE 802.11-based ad hoc networks which is able to differentiate QoS applications from Best Effort traffic. Through extensive simulations, we compare the performance of our proposal scheme with some others protocols like QPART for instance.
\end{abstract}

\section{Introduction}

The large availability of small wireless devices has enabled the deployment of mobile multihop wireless networks also called ad hoc networks. Ad hoc networks are autonomous and self-organized networks. They do not require any fixed infrastructure and nodes themselves solve topology changes due to mobility. This lack of centralized infrastructure makes the design of QoS protocols for these networks a challenging task. Moreover, due to their multihop and mobile features, QoS protocols must use distributed algorithms and not rely on global information.

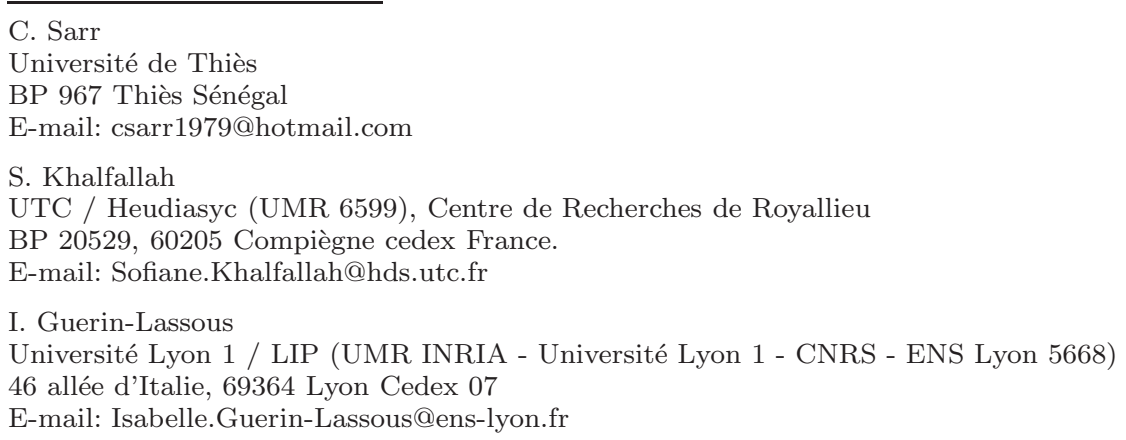


Quality of Service (QoS) focuses on several metrics like for instance delay, bandwidth, loss probability, etc. Our proposed scheme focuses on the bandwidth parameter, which is a basic metric often used to perform admission control, flow management or congestion control in ad hoc networks.

We assume that two types of applications are transmitted in the network:

- The first one requires guarantees on their throughput like video transmissions for instance. They are called QoS traffic henceforth.

- The second one is more tolerant to changes on their throughput like file transfer for instance. They are called Best Effort traffic henceforth or BE for short.

Many works focus on the guarantee of bandwidth for QoS traffic without dealing with Best Effort traffic. Most of these works in this area supply guarantees for QoS flows, thanks to an evaluation of the available bandwidth. However, these evaluations do not provide any differentiation between QoS and Best Effort data packets, which may lead to situations where there is not enough available bandwidth for a new QoS traffic just because most of the bandwidth is occupied by Best Effort traffic. Such approaches limit the number of accepted QoS flows.

A first solution could be to reserve a fixed proportion of the residual bandwidth for Best Effort traffic. However, such an approach is not efficient when few QoS flows are transmitted, since the throughputs of BE traffic are limited to a fixed value whereas they could have used the whole remaining bandwidth.

The main idea of our protocol (called DRBT for Dynamic Regulation of Best Effort Traffic) is to provide a QoS mechanism, which can regulate the throughput of Best Effort traffic (when it is necessary) and provides throughput guarantees to QoS flows, according to an evaluation of the available bandwidth. This evaluation mainly relies on the possibility for nodes to decode local information in order to differentiate QoS and Best Effort traffic and uses some mechanisms already defined in ABE [3].

The regulation scheme of DRBT proceeds in two phases:

- decreasing the throughput of Best Effort traffic in order to increase the number of accepted flows,

- increasing the throughput of Best Effort traffic to provide a maximal use of radio links when it is possible.

This regulation is coupled with a routing protocol in order to benefit of signaling packets to disseminate information required for our regulation mechanism. We have slightly modified AODV in order to transform it into DRBT. The remainder of this paper is organized as follows: Section 2 presents related work. Section 3 presents succinctly the different mechanisms of ABE used in DRBT. Section 4 describes our distributed protocol DRBT and finally simulations results are presented in Section 5.

\section{Related work}

To offer throughput guarantees to QoS flows, mobiles need first to evaluate the amount of bandwidth that is available in the network to ensure that the resource requirements of QoS admitted flows can be handled by the network. Different solutions have been proposed to evaluate the available bandwidth in IEEE 802.11-based multihop wireless networks, like for instance BRuIT [2], CACP [12] and ABE [3]. 
BRuIT and CACP attempt to provide a good estimation of the carrier sensing area in order to derive an accurate per node available bandwidth estimation. Indeed, with CSMA protocols (like in IEEE 802.11), two nodes within carrier sensing range share the medium and thus the bandwidth, even if they cannot directly communicate. Therefore, each node needs to know the channel occupancy in its carrier sensing area in order to derive an accurate available bandwidth estimation per node. ABE, as we will see in Section 3, adds a mechanism to estimate the synchronization of idle periods between emitter and receiver and an estimation of link probability of collision in order to provide an accurate available bandwidth estimation per link.

These different estimations are then used in a routing protocol in order to compute QoS routes, i.e. routes that offer at least the requested bandwidth. These works mainly focus on the QoS traffic and do not optimize the cohabitation between QoS flows and Best Effort flows. For instance, BRuIT allocates a small fixed share of the bandwidth to Best Effort flows, which is not very efficient when there is no QoS flow in the network. The permanent channel sensing realized by $\mathrm{CACP}$ or ABE may limit the number of accepted QoS flows. Indeed, with sensing, Best Effort flows are considered in the computation of the used bandwidth, which can lead to situations where most of the bandwidth is used by Best Effort flows and new QoS flows consider that there is not enough bandwidth to be transmitted. To summarize none of these protocols presented above deal with the presence of both QoS and Best Effort traffic.

In [4], a solution proposes to separate traffic by using two distinct channels of transmission. But this technique is difficult to implement and requires to change the current wireless cards.

SWAN [5] is a distributed protocol that dynamically guarantees bandwidth for QoS traffic without the use of QoS routing protocol. To achieve this goal, SWAN performs three mechanisms. An admission control mechanism and two regulation mechanisms, one for QoS flows and the other one for Best Effort traffic. The first admission control mechanism uses a passive approach to estimate the available bandwidth. Before each transmission of a QoS flow, a probe packet is sent from the sender towards the receiver to estimate the available bandwidth along this path. In function of this value, the admission control performed at the sender decides or not to send the QoS flow. There is no routing process because the route is supposed known. When SWAN estimates that the network is congested, the regulation mechanisms are started. The first mechanism of regulation handles QoS traffic. According to the modeling of the IEEE 802.11 DCF performed by Bianchi [6], available bandwidth can be evaluated. Consequently, as soon as a congestion is detected, the sender of the QoS flows simply reduces its throughput. The second mechanism of regulation concerns Best Effort traffic. The delay of ACK packets is measured. If this delay is higher than a predefined threshold, SWAN considers the network in a congested state and reduces the throughput of Best Effort flows.

The estimation of the residual bandwidth performed by probe packets sent from the sender to the receiver consumes an non-negligible amount of bandwidth. Moreover, for the first regulation mechanism, the assumptions of Bianchi's model are not valid in a multihop wireless environment. Therefore, the protocol SWAN provides an approximative residual bandwidth estimation, which makes more difficult the decision to regulate Best Effort traffic.

The protocol QPART [11] does not use any admission control protocol, but regulates the different flows according to a congestion threshold. The available bandwidth estimation is based on RED theory [8] which stipulates that to maintain the throughput constant over a multihop path, the queues size should be kept under a predefined 
threshold. If this threshold is exceeded, the regulation mechanisms are automatically activated. In case of congestion, the contention window size of Best Effort traffic in 802.11 is increased in order to make the transmission of QoS flows prior. QPART suffers from the same limitations as SWAN. Firstly, the available bandwidth based on RED theory is not adapted in the multihop wireless context where the presence of asymetric hidden terminals makes this theory inaccurate. Secondly, increasing the contention window size for Best Effort traffic just allows QoS data to have a probabilistic prior access on the radio channel.

To sum up, none of the described protocols in this section takes advantage of the differentiation between different kinds of traffic to provide an accurate regulation mechanism for the different traffic. In this work, we start with $\mathrm{ABE}$ that is, from our point of view, the most accurate protocol so far for evaluating the available bandwidth on a link, which is important for guaranteeing throughput. Then we add a differentiation mechanism to $\mathrm{ABE}$ in order to provide a more efficient bandwidth management. For instance, let's consider the scenario depicted on Figure 1. In this configuration, all the nodes are within communication range and the capacity (the maximum rate in the communication area) corresponds to $1600 \mathrm{~Kb} / \mathrm{s}$. Two flows are transmitted: a QoS flow of $500 \mathrm{~Kb} / \mathrm{s}$ and a Best Effort of $1000 \mathrm{~Kb} / \mathrm{s}$. A third flow attempts to transmit data on the medium.

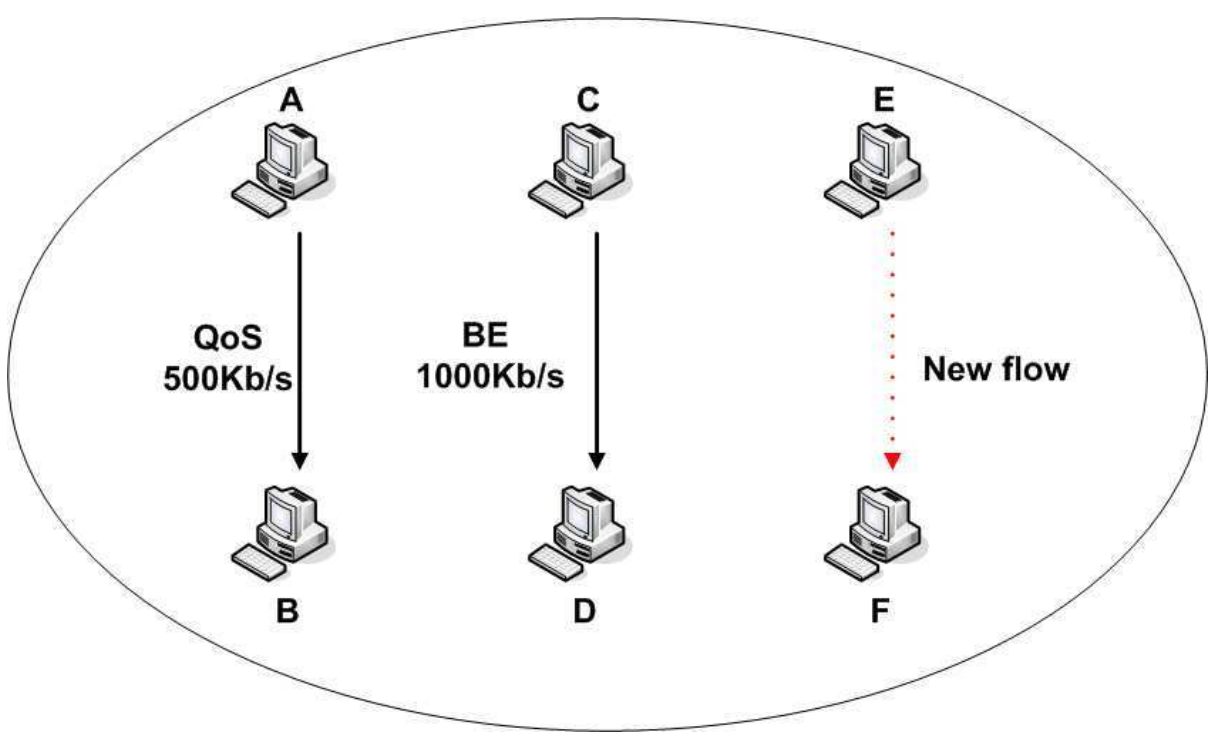

Fig. 1 Differentiation between QoS and Best Effort flow

With ABE, the perceived available bandwidth is almost null. Therefore this new flow can not be accepted. But, if we provide an estimation that differentiates QoS flows from Best Effort flows and that takes into account only transmissions of QoS flows in the evaluation, then we obtain a remaining bandwidth for the new flow almost equal to $1000 \mathrm{~Kb} / \mathrm{s}$. Therefore, the third traffic can be transmitted without degrading the existing QoS flow providing that a mechanism reducing the throughput of the 
Best Effort flow is used. Thus, we can accept more QoS flows, offer guarantees to the accepted QoS flows and provide the remaining bandwidth to the Best Effort flows.

Before describing the protocol DRBT, we present in the next section some mechanisms used in ABE to evaluate the available bandwidth of wireless links.

\section{$3 \mathrm{ABE}$}

For ensuring Best Effort traffic regulation, our solution relies on an accurate available bandwidth estimation. Hereafter, we define the available bandwidth between two neighbor nodes as the maximum throughput that can be transmitted between these two peers without disrupting any ongoing flow in the network. This term should not be confused with the link capacity (also called base bandwidth) that designates the maximum throughput a flow can achieve between two neighbor nodes, even at the cost of other flows' level of service degradation.

For the available bandwidth estimation, we choose the protocol ABE (Available Bandwidth Estimation), first proposed in [9] and then refined in [3]. In [3], the authors show that $\mathrm{ABE}$ is more accurate than several protocols with the same goal while requiring a small overhead. By considering the overlapping of the silence periods of both emitter and receiver of a link, the collision probability of the link and the backoff window size correlated to this collision probability, ABE reaches an accuracy in the estimation that is often not achieved by the other protocols.

As our regulation mechanism depends on this available bandwidth estimation, this section is devoted to the description of ABE. Of course, due to space limitation, we can not include all the details of ABE that is not the novelty of our proposition. The interested reader can refer to [3]. For providing an accurate evaluation, some phenomena need to be taken into account when the IEEE 802.11 MAC protocol operates:

- Carrier sense mechanism prevents two close emitters from transmitting simultaneously. Therefore, an emitter shares the channel bandwidth with all these close emitters. The channel utilization has to be monitored to evaluate the capacity of a node to emit a given traffic volume. As in many protocols, this channel utilization is computed by each node by monitoring the radio medium in its surroundings and measuring the total amount of time that is idle for emitting frames. Therefore, this method does not only take into account the bandwidth used in the transmission range of the nodes but also in the whole carrier sensing area.

- For a transmission to take place, both emitter and receiver need that no jamming occurs during the whole transmission. Therefore, the value of the available bandwidth on a link depends on both peers' respective channel utilization ratios but also on the idle periods synchronization. In [9], we propose a probabilistic method to estimate this synchronization. This estimation, for the link $(s, r)$, is denoted $E\left(b_{(s, r)}\right)$ in the following.

- Collision detection is difficult in a wireless environment. Therefore, whenever collisions happen, both colliding frames are completely emitted, maximizing the bandwidth loss. It is thus necessary to integrate this bandwidth loss in the available bandwidth estimation. In [3], we provide an estimation of the collision probability on each link. This estimation combines two approaches: i) A on line approach that computes the impact of the medium occupancy distribution at the receiver side thanks to the collision probability on Hello packets. These Hello packets are used in many ad hoc routing protocols and are required for computing the previous 
estimation $E\left(b_{(s, r)}\right)$ on each link; ii) A off line approach that takes into account the size of the packets sent by the source thanks to an interpolation. The goal of this last approach is to compute the collision probability that packets of known and fixed size will undergo on a link from the collision probability of Hello packets deduced from real measurements on the same link. This collision probability estimation is denoted $p$, in the following, and depends on the size of packets that will be sent.

- Finally, when collisions happen on unicast frames, the IEEE 802.11 protocol automatically retries to emit the same frame, drawing the backoff counter in a doublesized contention window. The time lost in additional overhead may also have an impact on the available bandwidth. In [3], we compute the mean backoff, $\overline{\text { backoff }}$ according to $p$ the collision probability computed in the previous estimation. It is then possible to deduce the proportion of bandwidth consumed by the backoff mechanism. This proportion is denoted by $K$ in the following and computed as $K=\frac{D I F S+\overline{\text { backoff }}}{T}$, where $T$ is the time separating the emission of two consecutive frames (see [3] for more details).

These different estimations are then combined to estimate the available bandwidth on a wireless link, i.e. between an emitter $s$ and a receiver $r$ :

$$
E_{\text {final }}\left(b_{(s, r)}\right)=(1-K) \cdot(1-p) \cdot E\left(b_{(s, r)}\right)
$$

Finally, the intraflow contention issue is also considered in ABE. The available bandwidth of link $(i, j)$ will take into account the neighboring hops in the flow path and will be reduced accordingly. See [3] for all the details.

\section{DRBT: a Dynamic Regulation protocol for Best Effort Traffic}

This section describes how we introduce the differentiation in the available bandwidth estimation and how we use this estimation for a regulation of Best Effort traffic. The first step is the differentiated estimation of the available bandwidth. It will allow us to quantify the proportion of the available bandwidth which is occupied by Best Effort flows. This estimation relies on the protocol ABE (Section 3). However, in the current state, ABE is not able to differentiate between QoS and Best Effort data packets. Therefore we present hereafter how we perform this differentiation in order to enhance the measurement accuracy.

\subsection{Differentiation between QoS and Best effort traffic}

As explained previously, a differentiation between QoS and Best Effort flows allows a better use of the available bandwidth for new QoS transmissions. We assume that each packet is marked in its IP header in order to know to which kind of flow it belongs, i.e. a QoS flow or a BE flow. The differentiation in the remaining bandwidth estimation is simply done at the MAC layer and consists in measuring only medium occupancy of QoS data packets during the monitoring phase of ABE.

Note that this differentiation is only possible if the node is able to decode data sensed over the medium since packets IP header has to be examined. Packets sent in the carrier sensing area of this node will not be decoded because the signal perceived 
is below the transmission range threshold. Consequently, in DRBT, the estimation of the available bandwidth is differentiated if the packet sensed over the medium can be decoded. In other words, Best Effort traffic that can not be decoded are included in the used bandwidth during the monitoring.

To summary, each node computes its differentiated remaining bandwidth by removing the bandwidth consumed by QoS flows in its communication range and consumed by all the flows in its carrier sensing area. Then this differentiated remaining bandwidth per node is used to compute the differentiated remaining bandwidth per link with the ABE method (Eq. (1)).

\subsection{Regulation of Best Effort traffic}

The previous available bandwidth estimation is not enough to provide guarantees to QoS flows. The BE traffic needs also to be regulated. In DRBT, the regulation scheme concerns only the Best Effort traffic. This regulation is done in two steps:

- Decreasing the throughput of Best Effort flows when a new QoS flow wishes to be transmitted and does not find enough available bandwidth because this one is partially consumed by Best Effort transmissions.

- Increasing the throughput of Best Effort flows when a QoS flow releases its bandwidth or moves to another transmission area.

This regulation is coupled with a routing protocol in order to benefit of signaling packets to disseminate information required for our regulation mechanism. We have slightly modified AODV in order to transform it into a QoS protocol, called DRBT henceforth. The choice of AODV is driven by the possibility to use broadcasted route request messages and route reply messages to inform and regulate $\mathrm{BE}$ flows and to find adequate (constrained) routes for QoS flows.

\subsubsection{Reduction of Best Effort traffic}

In this section we explain how we decrease the throughput of Best Effort flows. The regulation process of $\mathrm{BE}$ flows is triggered when a new QoS flow asks to be accepted in the network. Therefore, the search of an adequate route for a QoS flow is intimately linked to the possible regulation of some BE flows. To do this, DRBT does not introduce additional message overhead but uses classical RREQ (Route Request) and RREP (Route Reply) packets found in AODV. Every time a new QoS flow wants to transmit data, it ckecks the resources availability using these RREQ and RREP packets. The information stored on these packets with DRBT are:

- The throughput requested by the new QoS flow (ThroughputQoS).

- The number of Best Effort flows $(n b B E)$ within the neighborhood of the path on which the QoS flow is transmitted. For this, each Best Effort flow has a single identifier propagated on Hello messages. Therefore, each node can be able to know the number of Best Effort flows in its vicinity by analyzing these identifiers.

- The differentiated remaining bandwidth (DiffBandwidth) which only takes into account the QoS transmissions (when possible) as described in Section 4.1. 
The RREQ packet contains, in addition to the fields described previously, the address of the sender, the destination address and a sequence number. The sequence number is used in order to avoid cycles in the routing process, therefore a RREQ is just examined during its first passage. Each intermediate mobile that receives a RREQ performs an admission control by simply comparing whether the bandwidth requirement carried in the RREQ packet is lower than the differentiated available bandwidth of the link (previous sender, this node). If it is the case, the node updates $n b B E$ and DiffBandwidth (if necessary) and forwards the RREQ. Otherwise it discards it. $n b B E$ is incremented at each node by the number of Best Effort flows that the node knows. If the differentiated remaining bandwidth of the node that receives the RREQ is lower than the differentiated remaining bandwidth given in the RREQ, then the node modifies this field with its value. It allows us to know the available bandwidth computed along a path when considering only QoS transmissions (when possible).

When the destination receives a RREQ, it also needs to do the checking procedure as described above. Finally the destination sends a unicast route reply (RREP) to the initiator of the request along the reverse path to ensure that mobiles along the reverse path are still reachable.

Every time a Best Effort sender intercepts a RREQ or a RREP, it checks whether there is enough available bandwidth to carry the QoS flow without degrading it, by comparing its own throughput (ThroughputBE) with the parameter AvailableBandwidth computed as:

$$
\text { AvailableBandwidth }=\frac{\text { DiffBandwidth }- \text { ThroughputQoS }}{n b B E}
$$

If it is not the case, it reduces its throughput by sending a packet called DRP for Dynamic Regulation Packet. This packet, sent from the the IP layer towards the LL layer, activates the throughput reduction mechanism for Best Effort traffic. A leacky bucket algorithm is then applied, at the LL level, on the Best Effort packets with a rate corresponding to the AvailableBandwidth value ${ }^{1}$.

Equation 2 computes the new available bandwidth allocated to the Best Effort flow if the new QoS flow is accepted. This equation is conservative, because it considers that all the Best Effort flows, neighbors of a QoS flow on the path, share the same radio medium, which is not necessarily the case, and that it uses the minimum differentiated bandwidth on the path.

\subsubsection{Increase of Best Effort traffic}

When a QoS flow stops transmitting or moves to another transmission area, all the Best Effort flows that have reduced their bandwidth should increase their throughput to its initial value in order to use the maximum of the available bandwidth when possible. To address this issue, we use the Hello messages. Indeed, each node carrying a QoS flow encapsulates, in its Hello messages, information about the identifier of this flow and the differentiated available bandwidth.

\footnotetext{
1 Note that the throughput regulation is applied at the LL level. Packets may be dropped because the application rate is higher than the rate applied at the LL level. Regulate the rate at the application layer would remove this packet loss but implies another cross-layer interaction that we decided to not consider in this study and to let to future work.
} 
When a QoS flows stops transmitting or releases its bandwidth, it indicates this information in these Hello packets. The Best Effort emitter which is in the vicinity of this QoS flow will intercept these Hello messages indicating that a QoS transmission has stopped or that the available bandwidth has increased. Finally, the Best Effort flow can increase their throughput according to the updated available bandwidth. When a node moves, the nodes carrying a Best Effort flow do not received Hello messages anymore and can thus use their previous allocated bandwidth providing that they have stored it, according to this updated available bandwidth.

\section{Simulations}

In this section, we evaluate the performances of DRBT and compare it with other approaches. We use the network simulator $2(\mathrm{NS}-2.27)^{2}$ and the IEEE 802.11 implementation provided with the simulator. The parameters used for all scenarios are presented on Table 1, except when specified. We use different physical rates in different scenarios in order to test the effectiveness of our approach under different physical rates.

We compare the performance of our regulation mechanism through the DRBT protocol described above with AODV [1], ABE [10] and QPART [11]. We chose QPART because as far as we know, it is one of the most recent solutions that provide both differentiation between QoS and Best Effort in addition to a regulation scheme as DRBT. The comparison between DRBT and ABE will allow us to evaluate the added value of a differentiation between QoS and Best Effort data packets. We also compare DRBT with AODV as a baseline for comparison.

\begin{tabular}{|c|c|}
\hline Parameters & Values \\
\hline \hline HELLO interval & $1 \mathrm{~s}$ \\
\hline Packet size & 1000 bytes \\
\hline Physical rate & 2 or $11 \mathrm{Mb} / \mathrm{s}$ \\
\hline Communication range & $250 \mathrm{~m}$ \\
\hline Carrier sensing range & $550 \mathrm{~m}$ \\
\hline Grid size & $1000 \times 1000 \mathrm{~m}$ \\
\hline LL queue size & 100 packets \\
\hline
\end{tabular}

Table 1 General parameters for simulations

\subsection{First topologies}

\subsubsection{A simple scenario}

For this simulation, we consider a simple topology of two pairs of nodes within the same communication range as depicted on Figure 2.

At the date $\mathrm{t}=1 \mathrm{~s}$, a Best Effort connection of throughput $1000 \mathrm{~Kb} / \mathrm{s}$ is established between A and B. Four seconds later, a new QoS flow of throughput $1000 \mathrm{~Kb} / \mathrm{s}$ is also established between $\mathrm{C}$ and $\mathrm{D}$. At date $\mathrm{t}=30 \mathrm{~s}$, the QoS flow stops its transmission. The

\footnotetext{
2 http://www.isi.edu/nsnam/ns/
} 


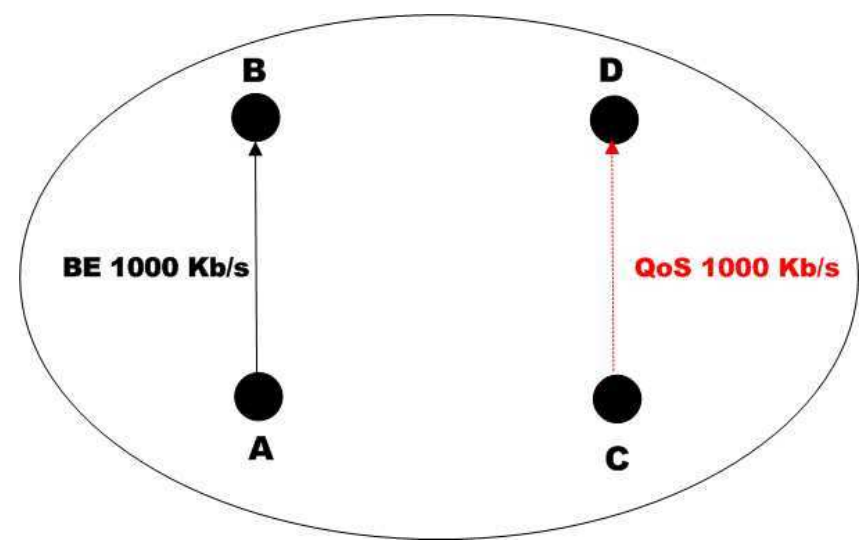

Fig. 2 Two pairs

simulation lasts fifty seconds and the capacity is fixed to $1,6 \mathrm{Mb} / \mathrm{s}$ (with a physical rate of $2 \mathrm{Mb} / \mathrm{s})$.

When AODV is used, as shown on Figure 3(a), each connection tries to send its data packets when possible without any regulation mechanism and this situation leads to a shared medium between the Best Effort and the QoS flows. The throughput of these two flows are consequently degraded.

When ABE is performed (Figure 3(b)), the admission control step estimates that there is not enough available bandwidth to carry the QoS flow with its bandwidth requirement. Hence, only Best Effort traffic is transmitted.

QPART is not able to enough decrease the throughput of the Best Effort flow. Therefore, the throughput request is not guaranteed to the QoS flow, as shown in Figure $3(\mathrm{~d})$.

With DRBT, the decrease is more accurate and consequently, the QoS flow is carried with its bandwidth requirement without any degradation, as shown in Figure 3(c).

\subsubsection{A random topology}

To compare the different protocols and illustrate the effectiveness of DRBT to provide a better regulation mechanism, we generate random topologies with random constant bit-rate flows (random source, random destination and random throughput with fixed 1000 bytes frames). For each of these protocols, similar scenarios (same number of nodes and same number of flows) lead to similar behaviors. The scenario consists of a static network involving 10 randomly positioned nodes. Two QoS and three Best Effort CBR connections and established. The physical rate is set to $2 \mathrm{Mb} / \mathrm{s}$. Table 2 sums up the throughputs requested by all flows in the network.

As in the previous simulation, the same observations can be done. AODV (Figure 4(a)) involves a sharing of resources among the different flows without any priority for QoS flows. ABE estimates that there is not enough available bandwidth to carry QoS flows with their bandwidth requirements. Hence, only Best Effort traffic are transmitted (Figure 4(b)).

QPART is not able to enough decrease the throughputs of the Best effort flows. Therefore, QoS flows are degraded as shown in Figure 4(d). 


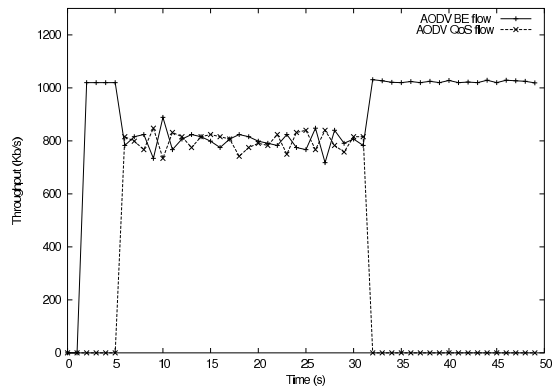

(a) AODV

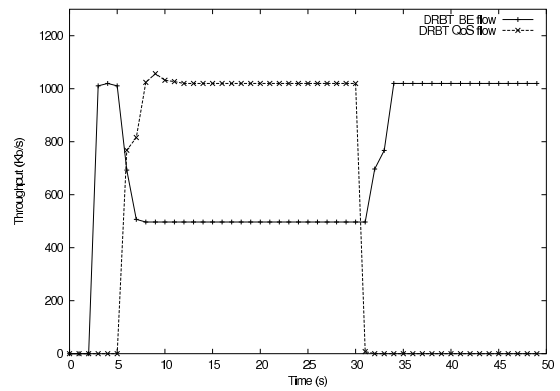

(c) DRBT

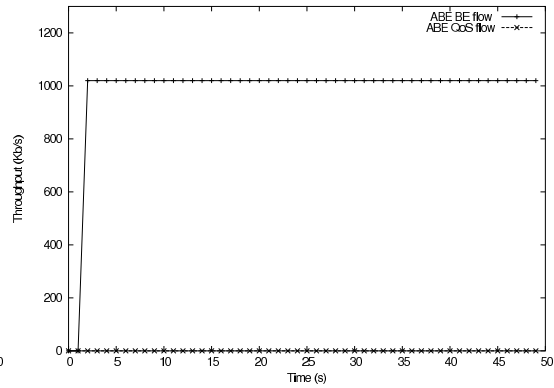

(b) $\mathrm{ABE}$

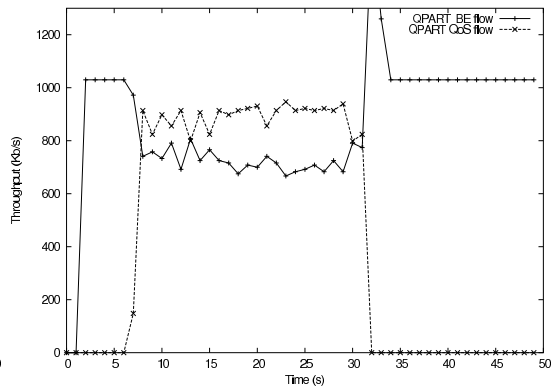

(d) QPART

Fig. 3 Throughputs obtained with AODV, ABE, DRBT et QPART

\begin{tabular}{|c|c|c|c|}
\hline Flow & Type & Desired throughput (Kb/s) & Date of emission (s) \\
\hline \hline CBR1 & Best Effort & 319 & 10 \\
\hline CBR2 & Best Effort & 164 & 20 \\
\hline CBR3 & Best Effort & 386 & 30 \\
\hline CBR4 & QoS & 129 & 40 \\
\hline CBR5 & QoS & 281 & 50 \\
\hline
\end{tabular}

Table 2 Throughputs desired by all flows

Finally, DRBT reduces effectively the throughputs of the Best Effort flows so that the QoS flows are carried with their bandwidth requirements without any degradation.

\subsection{Accuracy of the regulation mechanism of DRBT}

Let us now investigate the general case. To reflect the accuracy of DRBT, we define a new metric accounting for the number of right admissions. A right admission happens when the admission control protocol allows the routing of a QoS flow and this flow's throughput is not degraded by more than $5 \%$ when it gets transfered. This definition implies that the differentiated available bandwidth estimation and the admission control are reliable. The acceptance ratio is defined by the following expression: 


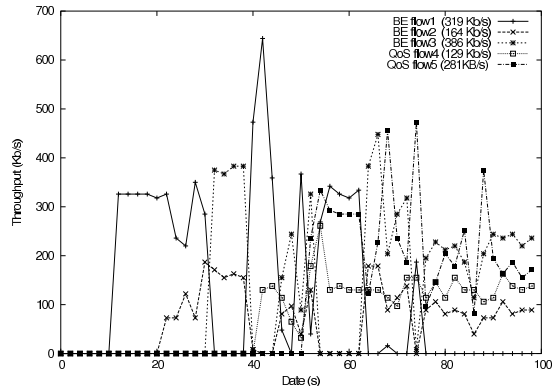

(a) AODV

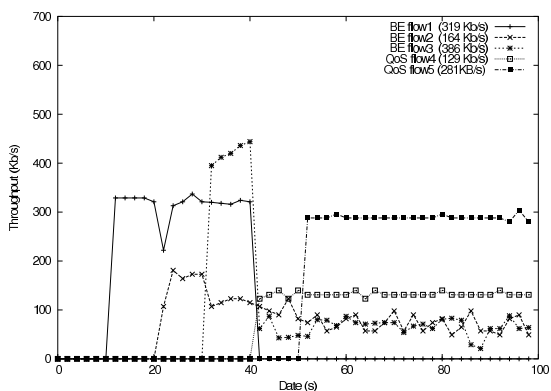

(c) DRBT

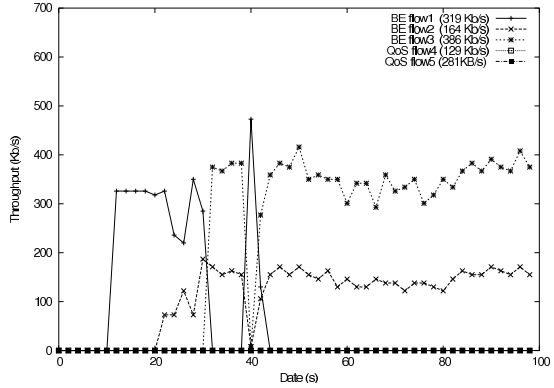

(b) $\mathrm{ABE}$

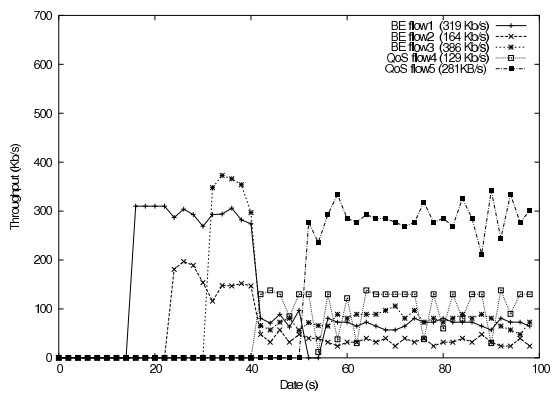

(d) QPART

Fig. 4 Throughputs obtained with AODV, ABE, DRBT et QPART in a random topology

$$
\beta=\frac{\text { Number of right admission }}{\text { Number of flows requesting QoS routes }}
$$

A falsely admitted flow either degrades the throughput of close flows or is not able to achieve its desired throughput. Hence, the value of $\beta$ decreases. So $\beta$ is able to characterize as well an under-estimation as an over-estimation.

We measured the value of $\beta$ by simulation on networks composed of 10 to 40 nodes, using an $11 \mathrm{Mb} / \mathrm{s}$ medium capacity. Each simulation lasts 100 seconds and 15 randomly chosen pairs of nodes try to establish CBR connections (5 Best Effort connections and 10 QoS connections). Each flow starts according to the Poisson model with average arrival rate of one flow every 5 seconds. The throughput of each connection is uniformly drawn between $0 \mathrm{~kb} / \mathrm{s}$ and $300 \mathrm{~kb} / \mathrm{s}$. Results presented are the average of 30 simulations for a defined number of nodes with a confidence interval of $95 \%$.

Influence of the used protocol: Figures $5(\mathrm{a})$ represents the values of $\beta$ for AODV, ABE, QPART and DRBT in function of the number of nodes in the network.

When the network is not too dense (between 10 and 20 nodes), the acceptance ratio of DRBT is high (about 60\%) while this value is lower for QPART (about 41\%). Therefore, the differentation between QoS and Best Effort data packets and the regulation scheme provided by both QPART and DRBT allows these two protocols to have better performances than ABE and AODV. 


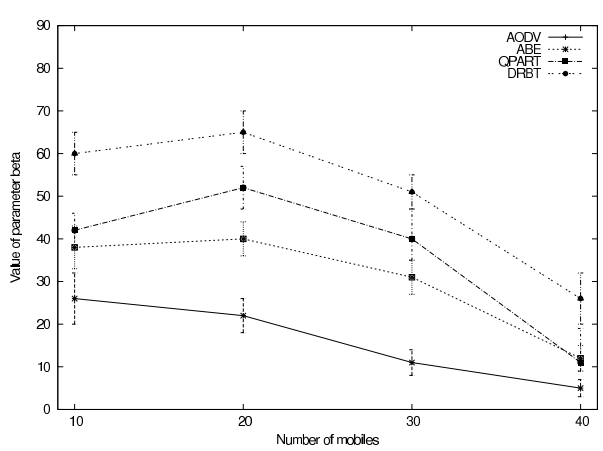

(a) Carrier sensing range $=2 \times$ communication range

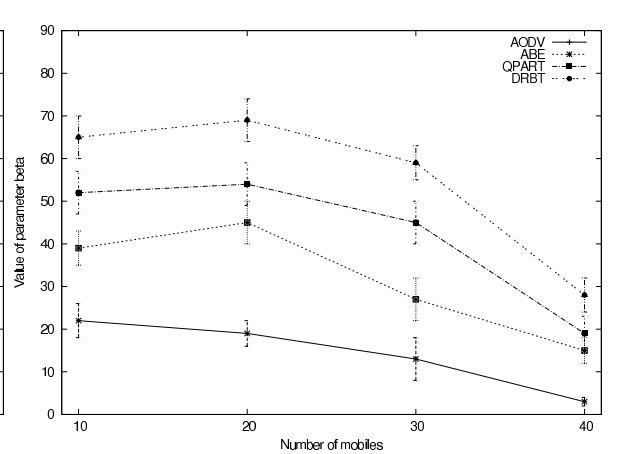

(b) Carrier sensing range $=$ communication range

Fig. 5 Acceptance ratio of QoS flows with AODV, ABE, QPART and DRBT

When the network becomes denser (between 20 and 30 nodes), $\beta$ decreases, which is expected as the available bandwidth per link decreases and lower quality routes are established. However, DRBT can also transmit about $51 \%$ of QoS flows.

Finally, when the network becomes very dense (between 30 and 40 nodes), the residual bandwidth becomes low and even a reduction of throughput of Best Effort traffic can not release enough available bandwidth to allow QoS flows to be transmitted with their throughput requirements. Nevertheless, DRBT still correctly transmits about $29 \%$ of QoS flows while all the others protocols transmit in the best case almost than $11 \%$.

Influence of the carrier sensing range: For this simulation, we reduce the carrier sensing range until it is equal to the communication range. Results are presented in Figure 5(b). The acceptance rate of DRBT is higher than previously for a same number of nodes and is always higher than for QPART, ABE and AODV. In fact, reducing the carrier sensing range allows DRBT to decode more information stored on packets. This facilitates the differentiation between QoS and Best Effort packets in DRBT, and thus the regulation of more Best Effort flows. On the other hand, the disadvantage of reducing the carrier sensing is that more hidden terminals configurations are created which poses problems to AODV, since it does not take into account in their measurement configurations like hidden terminals contrary to DRBT.

\subsection{Loss rate}

Other parameters like loss rate or delay are of some importance for QoS applications. We have focused our study on the loss rate parameter because this parameter seems to be more critical than delay in multihop wireless networks of medium size (see [7]). We study the loss rate on QoS flows obtained with AODV, QPART and DRBT. We have tested the same scenarios than the ones tested in Section 5.2 with a carrier sensing range two times larger than the communication range. In Figure 6, we see that loss rate is smaller with DRBT than with AODV and QPART. For instance, with 40 nodes, this packet loss with DRBT is two times smaller than QPART and three times smaller 
than AODV. This is mainly explained by the fact that with DRBT, more QoS flows are transmitted with less packet losses thanks to a good estimation of the available bandwidth and to a better regulation of close Best-effort flows.

Note that, however, the values obtained on loss rate are still high, even with DRBT, for some QoS applications. It seems that a smart bandwidth management decreases the loss rate but is not enough to achieve small target loss rates required by some applications.

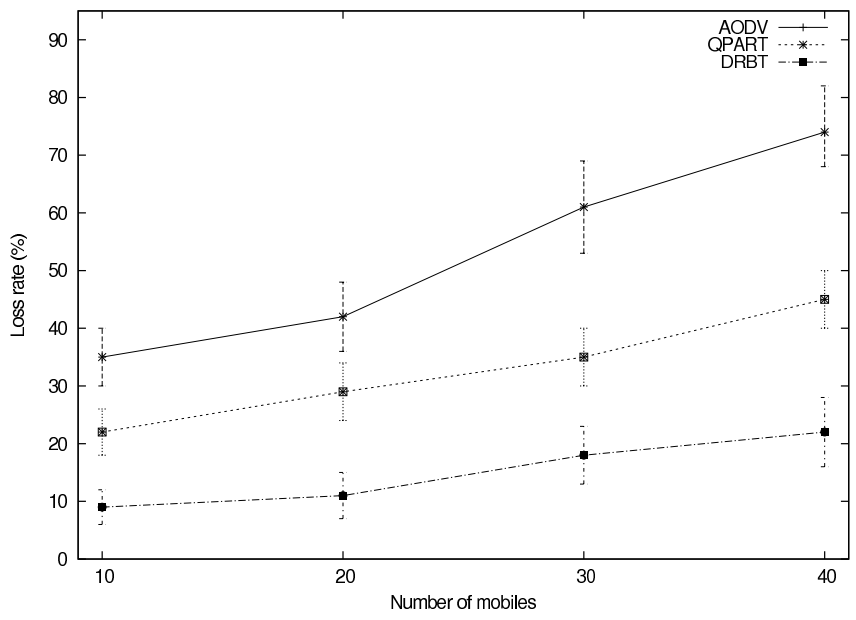

Fig. 6 Loss rate on QoS flow packet with AODV, QPART and DRBT

\subsection{Signaling overhead}

We study the signaling overhead induced by the use of the broadcast technique of AODV in which we have added new fields in the control packets. We have tested the same scenarios than the ones tested previously. Figure 7 shows the signaling overhead with the control packets RREQ, RREP and RRER (when there is an error at the routing layer) in bytes of DRBT and AODV. Even though larger control packets are used with DRBT compared to AODV (e.g. 48 bytes for RREQ packets or 68 bytes for RREP packets with DRBT versus 32 bytes for RREQ and RREP packets with AODV), the signaling overhead in bytes is much smaller with DRBT than with AODV. For instance, with 40 nodes, this signaling overhead is three times smaller. This is mainly explained by the fact that with DRBT, less RREQ packets are forwarded due to the bandwidth requests of the QoS flows. It is also interesting to note that the number of RRER packets is much less with DRBT than with AODV. For instance, with 40 flows, there are around 200 RERR packets compared to the 4800 RERR packets with AODV. It tends to show that DRBT has an efficient bandwidth management which results in less collisions and thus in less route recoveries than with AODV. 


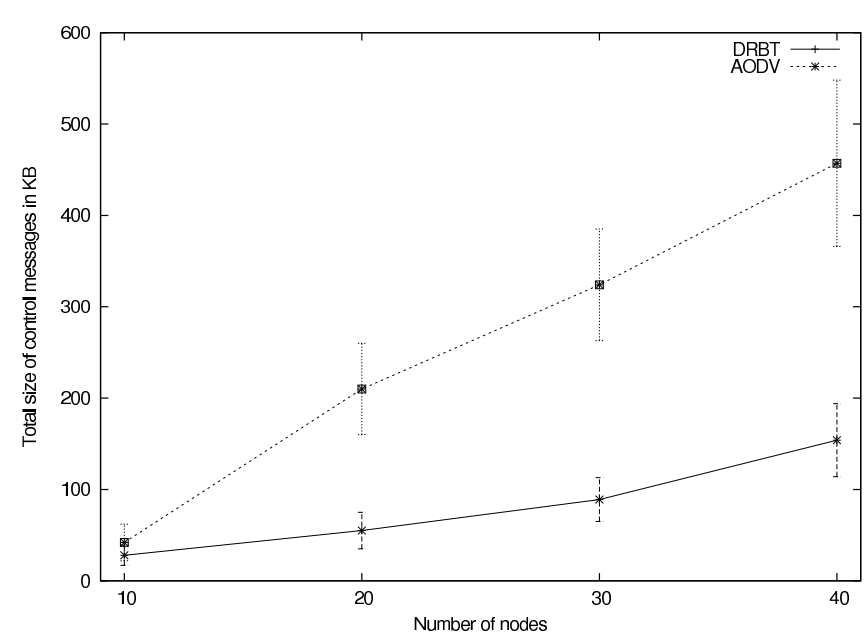

Fig. 7 Signaling overhead in bytes with AODV and DRBT

5.5 Influence of Best Effort traffic profile

So far, only CBR traffic has been used for Best Effort traffic in the previous simulations. In this section, we use VBR (Variable Bit Rate) traffic for Best Effort traffic. The scenario consists of a static network involving 10 randomly positioned nodes. Two QoS and three Best Effort VBR connections are established. The physical rate is set to $2 \mathrm{Mb} / \mathrm{s}$. The used VBR traffic has an exponential distribution for the burst and idle periods, with a mean idle period of $100 \mathrm{~ms}$ and a mean burst period of $150 \mathrm{~ms}$. Table 3 sums up the throughputs requested by all flows in the network.

\begin{tabular}{|c|c|c|c|}
\hline Flow & Type & Desired throughput (Kb/s) & Date of emission (s) \\
\hline \hline VBR1 & Best Effort & 266 & 10 \\
\hline VBR2 & Best Effort & 268 & 20 \\
\hline VBR3 & Best Effort & 258 & 30 \\
\hline CBR1 & QoS & 315 & 40 \\
\hline CBR2 & QoS & 591 & 50 \\
\hline
\end{tabular}

Table 3 Throughputs desired by all flows

The same remarks as the ones provided in Section 5.1.1 can be done: AODV involves a sharing of resources among the different flows without any priority for QoS flows (Figure 8(a)) ; ABE prevents one QoS flow from being transmitted because it estimates that there is not enough available bandwidth (Figure 8(b)) ; QPART is less effective than with CBR BE flows (Figure $8(\mathrm{~d})$ ). It seems that the alternance of burst/idle periods prevents QPART from adapting and converging towards the right size of the congestion window of each kind of traffic ; on the other hand, DRBT reduces effectively the throughputs of the Best Effort flows so that the QoS flows are transmitted with their bandwidth requirements without any degradation. Compared to the results of Section 5.1.1, the throughputs of the QoS flows with DRBT are more variable. This is probably due to the variations of the BE traffic that imply variations in the remaining 


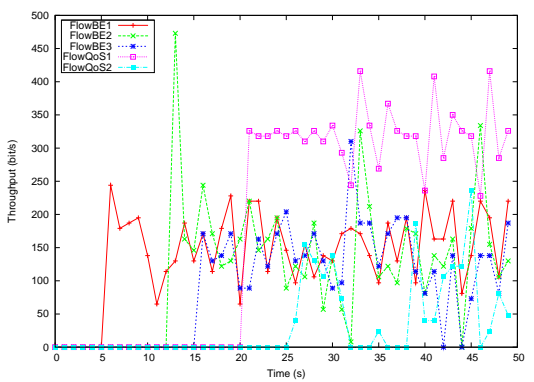

(a) AODV

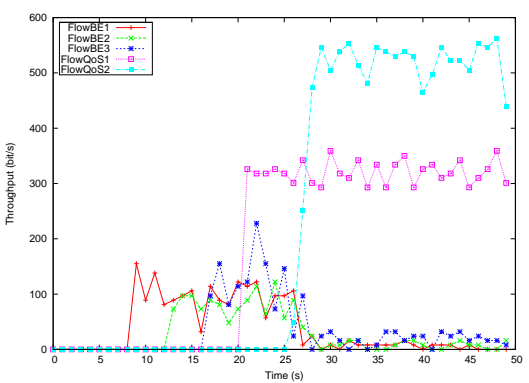

(c) DRBT

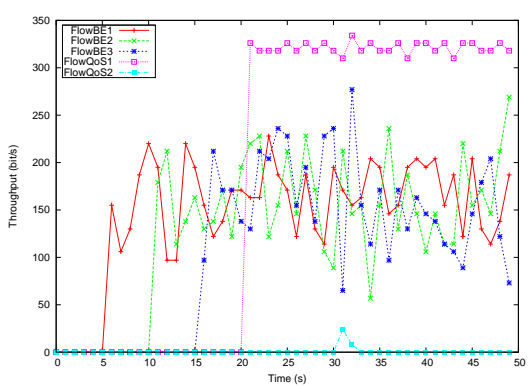

(b) $\mathrm{ABE}$

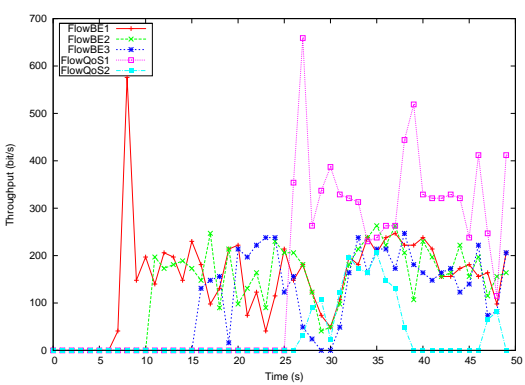

(d) QPART

Fig. 8 Throughputs obtained with AODV, ABE, DRBT et QPART in a random topology with VBR BE traffic

bandwidth not taken into account in the remaining bandwidth estimation computed during the establishment of the QoS flows (RREQ and RREP phases).

\section{Conclusions and future works}

In this paper, we have presented DRBT (Dynamic Regulation of Best Effort Traffic), a protocol which guarantees bandwidth of QoS flows by adpating effectively and dynamically the throughputs of Best Effort transmissions when it is necessary. Our protocol relies on an estimation of the available bandwith differentiated according to the type of packets (QoS or Best Effort data packets). With these features, DRBT increases the acceptance ratio of QoS flows, while providing a better usage of the radio medium. Furthermore, the effectiveness of our protocol is shown through simulations, where DRBT effectively manages the throughputs of QoS transmissions by dynamically adapating rate of close Best Effort traffic, compared to other protocols like AODV, ABE and QPART. We show also that DRBT reduces the loss rate compared to the other tested protocols. However, the values achieved may be still too high for some QoS applications. 
In future works, we intend to improve some features of DRBT and more particularly to deal with the problems induced by the carrier sensing mechanism. We also plan to design a smarter share of the medium between Best Effort flows. Indeed, the current share is very conservative since it considers that all the BE flows neighbors of a QoS flow share the same medium, which it may not be the case. Therefore, more capacity could be used for BE flows. Another important point is to deal with mobility which prevents from guaranteeing resources due to the disappearance of link radio.

\section{References}

1. Charles E. Perkins, Elizabeth M. Belding-Royer and Samir Das. Ad hoc On-Demand Distance Vector (AODV) Routing. Internet Request For Comments RFC 3561. Internet Engineering Task Force IETF, July 2003.

2. C. Chaudet and I. Guérin Lassous. BRuIT - Bandwidth Reservation under InTerferences influence. In In Proceedings of European Wireless 2002 (EW2002), Florence, Italy, Feb 2002.

3. Cheikh Sarr, Claude Chaudet, Guillaume Chelius and Isabelle Guérin-Lassous. Bandwidth Estimation for IEEE 802.11-Based ad hoc network. IEEE Transactions on Mobile Computing, 7(10), October 2008.

4. Fethi Filali. Towards a fully distributed QoS-aware MAC protocol for multihop wireless networks. In IWWAN 2005, International Workshop on Wireless Ad-hoc Networks, May 23rd - 26th 2005, London, UK, May 2005.

5. Gahng-Seop Ahn, Andrew T. Campbell, Andreas Veres and Li-Hsiang Sun. SWAN: Service Differentiation in Wireless Ad Hoc Networks. In IEEE INFOCOM, 2002.

6. Giuseppe Bianchi. Performance Analysis of the IEEE 802.11 Distributed Coordination Function. IEEE Journal on Selected Areas in Communications, Volume 18(3):pages 535547, March 2000.

7. H. Pucha, S. M. Das, and Y. C. Hu. The performance impact of traffic patterns on routing protocols in mobile ad hoc networks. Computer Networks, 51(12), 2007.

8. Sally Floyd and Van Jacobson. Random Early Detection Gateways for Congestion Avoidance . IEEE/ACM Transaction on Networking, 1:397-413, 1993.

9. C. Sarr, C. Chaudet, G. Chelius, and I. Guérin-Lassous. A node-based available bandwidth evaluation in ieee 802.11 ad hoc networks. International Journal of Parallel, Emergent and Distributed Systems, 21(6), 2006.

10. C. Sarr, C. Chaudet, G. Chelius, and I. Guérin Lassous. Improving Accuracy in Available Bandwidth Estimation for 802.11-based Ad Hoc Networks. Technical Report 1, INRIA, June 2007.

11. Yaling Yang and Robin Kravets. Distributed QoS Guarantees for Realtime Traffic in Ad Hoc Networks. In IEEE International Conference on Sensor and Ad Hoc Communications and Networks (SECON), pages 118-127, Oct 2004.

12. Y. Yang and R. Kravets. Contention Aware Admission Control for Ad Hoc Networks. IEEE Transactions on Mobile Computing, 4(4):363-377, 2005. 